\title{
Teorías de las organizaciones: panorama actual
}

Jorge Dávila*

\section{Resumen}

Se propone una comprensión del debate contemporáneo sobre el status epistemológico de las teorías de organizaciones. Para ello, se hace un seguimiento de la revisión y proposiciones que en torno a ese debate realiza $M$. Reed. El modo como este autor ha formulado el problema que representa para los académicos el estado de dispersión en que se encuentra el campo de estudio del fenómeno organizacional, abre una interesante potencialidad para encauzar ese debate de manera que sea posible vislumbrar una práctica, al menos académica, más acorde con un ejercicio razonable del pensamiento. El propósito y conclusión de este trabajo es estimular la referida potencialidad.

Palabras clave: Teoría de organizaciones, epistemología, práctica académica, técnicas gerenciales

\section{Organizational Theories: Contemporary View}

\section{Abstract}

A comprehension of the current debate regarding the epistemological status of Organizational Theories is presented. The comprehension is reached through a critical review of M. Reed's arguments concerning the epistemological disorder in the field of Organizational Theories. Reed's contributions provides a potential way for the constitution

\section{Recibido: 02-10-97 . Aceptado: 19-12-97}

* Miembro del Departamento de Sistemología Interpretativa de la Universidad de Los Andes (Mérida 5101 - Venezuela; fax: 58-74-402997). Es Protesor Asociado con estudios de postgrado en Ciencias Sociales (EHESS, París) y se dedica al estudio de instituciones públicas y del problema de la dimensión histórica en la teoría interpretativa de sistemas. 
of a practice (at least in academic milieu) grounded on a reasonable exercise of thought. The purpose and conclusion of this paper is to enhance the potentiality of the path suggested by Reed.

Key words: Organizational theory, Epistemology, Academic practice, Managerial techniques.

\section{Introducción}

Todo aquel que se haya paseado por el campo de estudio de las organizaciones debe haber sentido la impresión de un vacio de fundamentación. En efecto, en cualquiera de las expresiones que dicho campo ha tenido, y tiene (por ejemplo, y sólo por citar algunas: teoría administrativa, ciencia gerencial, arte de dirigir, sistemas organizativos, sociologia de organizaciones, psicología industrial) se dice, de manera explícita o implicita, que o bien no hay modo de justificar los presupuestos últimos que alli se asumen, o bien que tales presupuestos están fuera del alcance de la propia expresión del campo de estudio de las organizaciones.

Ciertamente que semejante vacio, o verrtigo que el estudioso puede sentir, no ha limitado la proliferación de enfoques o paradigmas del estudio de organizaciones. Por el contrario, esa proliferación contribuye con la afirmación de la aparente imposibilidad de hacer descansar al campo teórico del estudio de organizaciones en un sólido piso. Pero, más interesante, ese vacio tampoco ha podido evitar la proliferación de las discusiones en torno al status epistemológico de esos enfoques o paradigmas.

Se puede afirmar que quienes nos dedicamos, académicamente o no, al estudio del comportamiento de organizaciones tenemos una fuerte tendencia a convivir con esta situación en la que se conjuga el vacio de fundamentación con las referidas proliferaciones. Esa tendencia la alimentamos con nuestra misma práctica, especialmente la académica, al acostumbrarnos a saltar, por sobre el vacío, de torre en torre (de enfoque en enfoque), evitando el vértigo $\mathrm{y}$, al mismo tiempo, redescubriéndolo.

En este trabajo se pretende contribuir con la limitación de esa tendencia. Se ofrece un panorama que permite descender de cualquier torre de manera que en el descenso se pueda ir viendo no tanto el piso que sostiene las torres como la necesidad de contribuir a que, efectivamente, haya un piso. De modo que, con esta contribución el lector está llamado a ser uno más que descubra que la convivencia con el vértigo, si es que tiene que ser irremediable, al menos pueda experimentarse como terminando en un piso y no como una espiral sin fin. Como veremos, esta exigencia parece ser insoslayable para la práctica académica del estudio de las organizaciones.

El panorama que aquí se presenta constituye, básicamente, una revisión de dos recientes trabajos de Michael Reed sobre el tema. Ellos son: el libro titulado The Sociology of Organizations. Themes, Perspectives and Prospects (Reed, 1992), y el capítulo ti- 
tulado Organizations and Modernity:Continuity and Discontinuity in Organization Theory en el libro editado por J. Hassard y M. Parker con el título de Postmodernism and Organizations (Reed, 1993).

El trabajo de M. Reed se propone, a mi modo de ver, esencialmente dos objetivos estrechamente conectados. Por una parte, criticar el encajonamiento en que se encuentra la discusión epistemológica sobre la Teoría y la Sociología de Organizaciones y, por otra parte, proponer la estructuración del campo académico relativo al estudio de organizaciones sobre la base de una cierta manera de concebir el fenómeno organizacional. En las siguientes secciones nos referimos en detalle a cada uno de esos objetivos.

\section{El encajonamiento del debate epistemológico sobre las teorias de organizaciones}

En la Teoría de Organizaciones estamos viviendo "un tiempo en el que la definición tanto del objeto de estudio (subjet matten como de las reglas para la construcción de la teoria y de la práctica de investigación están abiertos al debate y a interpretaciones conflictivas" (Reed, 1992: 255). Esta afirmación de Reed es propia de la actualidad del desarrollo del estudio de organizaciones, si por este último se entiende el desarrollo del "campo académico" donde se plantean, con cierto rigor, preguntas relativas a la validez del contenido, método y fundamentación del estudio del 'fenómeno organizacional". Esa actualidad es vista por el autor según una doble caracterización: una cronológicamente, data de las últimas tres décadas (a esto nos referimos en de- talle más adelante); otra, en los últimos diez años se presenta como una suerte de encajonamiento que intentamos aclarar enseguida.

El debate sobre los estudios de organizaciones se suele presentar como sujeto a la escogencia de una de estas tres posibilidades:

a. El relativismo absoluto (el autor le llama "anything goes") propio del criterio de selección metódica que escoge por conveniencia la combinación metódica apropiada para el "problema" que se estudia. No creo que resulte exagerado ubicar aquí la tendencia predominante en los estudios llamados "sistémicos" al estilo del Critical Systems Thinking - Total Systems Intervention (Jackson, 1992; Flood and Jackson, 1992) o el uso simultáneo de las "metáforas" de Gareth Morgan (Morgan, 1986; 1989).

b. El desarrollo múltiple e irreconciliable de varias teorias. En este caso se supone que en el nivel de fundamentación de la teoría aparecen supuestos que, siendo tan contrarios, no pueden alcanzar a reunirse en un debate. El ejemplo prototípico es la inconmensurabilidad paradigmática de los cuatro tipos de fundamentación sociológica expresados en el mapa de clasificación (de paradigmas sociológicos y teorias de organizaciones) de G. Burrell y $\mathrm{G}$. Morgan (Burrell and Morgan, 1979).

c. La "vuelta a lo básíco"; es decir, la aceptación de que todos los desarrollos (metódicos y de fundamentación) que pretendieron romper con el desarrollo teórico dominante por he- 
rencia en el estudio de organizaciones - la llamada ortodoxia funcionalistadeben rendir su bandera, después de tantos traspiés, a un estructuro-funcionalismo renovado con las pocas afirmaciones positivas que fuera de su dominio se ha logrado. Este es el caso de las proposiciones de L. Donaldson (Donaldson, 1985). Aquí puede ubicarse la tendencia dominante del "enfoque de sistemas" que supone a las organizaciones como sistemas sociotécnicos; es decir, como instrumentos racionales de coordinación y control adecuados a las exigencias ambientales y a los imperativos funcionales que éstas le imponen. El modelo de la "teoria de la contingencia", repotenciado en sus subsistemas funcionales con el conocimiento parcial obtenido por otras investigaciones, sería la piedra angular del análisis organizacional.

¿En qué consiste, entonces, el encajonamiento? En que estas tres posibilidades parecen mostrar un agotamiento definitivo de la característica del campo académico del análisis organizacional; a saber, "la apertura al debate y al conflicto de interpretaciones". En otras palabras, parece que desaparece una especie de asiento común para el debate. Pero, ¿es que ha habido tal asiento común? Hagamos un pequeño tour por la primera característica de la "actualidad" (su ubicación cronológica en las últimas tres décadas) para ser más precisos en relación con el referido agotamiento.

En términos generales suele aceptarse que la constitución de un campo académico en relación con el estudio de organizaciones no aparece sino hasta mediados de este siglo. Esa aceptación supone que el desarrollo desde inicios de siglo (momento en que suele ubicarse la preocupación inquisitiva por el fenómeno organizacional: la organización productiva del trabajo fabril como tema de interés para las nacientes "ciencias administrativas" y para la ingeniería industrial) vino a converger en una manera única de concebir el estudio de organizaciones que reunia la 'decantación' de los aportes del "taylorismo", de la "escuela de relaciones humanas" y del "análisis de toma de decisiones" propio desarrollado desde los años cincuenta. Esa única manera de concebir el estudio de organizaciones encontró en el lenguaje sistémico, propio de la sociología estructuro-funcionalista y de la "teorla general de sistemas", su nicho perfecto. $M$. Reed denomina a esta concepción como el "marco analítico" de las "organizaciones como sistemas sociales". En ese marco se definen las organizaciones como "unidades sociales dirigidas a la prosecución de metas colectivas o al cumplimiento de necesidades institucionales para la sociedad o el ambiente del que ellas son parte constitutiva" (Reed, 1992: 75-76).

Es frente a esta concepción única $y$, por ende, dominante, que van a surgir, a partir de la década de los años sesenta, otras concepciones como por negación, o más bien oposición, al modelo estructuro-funcionalista-sistémico. Así se define claramente el piso común del debate, aun cuando éste no se presenta, inicialmente, más que como pura oposición, bien que el ałán intelectual sea meramente empírico o cargado de cierta reflexión teórica. Así se verán surgir otros "marcos anallticos" que Reed denomina: "organizaciones como 
orden negociado", "organizaciones como estructuras de poder y dominación" u "organizaciones como construcciones simbólicas" (Reed, 1992: 75-112).

Esa proliferación e irrupción de otros marcos de análisis (es decir, su variedad) exige más precisión a la caracterización del piso común del debate. Reed ve en el trabajo de David Silverman (Silverman, 1970) la expresión precisa del debate: la contrapuesta de un "marco de referencia" basado en la "Iógica de la acción" frente al dominante "marco de referencia" que descansa en la "bógica del sistema" (Reed, 1993: 172). Con base en este piso común para el debate epistemoIógico la proliferación de marcos analíticos, métodos y técnicas de investigación se encamina en una combinación de investigaciones específicas de fenómenos organizacionales concretos y reflexiones de corte epistemológico. Puede decirse que la expresión de este piso común, dada su expresión negativa, es una primera forma del encajonamiento; pero, entiéndase bien, un encajonamiento que resulta fructífero por ambos lados del desarrollo del campo académico; la discusión epistemológica y el avance del propio análisis organizacional. Así no resultará extraño encontrar, mediando los años setenta y ochenta, análisis organizacionales empíricos que hacen descansar su fundamentación teórica en, por ejemplo, la filosofia fenomenológica, como fue el caso para los estudios denominados etnometodológicos, o bien encontrar justificaciones del traspaso de hallazgos teórico-empiricos propios del estudio antropológico de culturas donde no se manifiesta el fenómeno organizacional propiamente dicho, al análisis de mani- festaciones del manejo o construcción simbólico de la vida organizacional.

De este modo el piso común comienza a exigir mayor precisión. La mera contraposición a la concepción estructuro-funcionalista-sistémica da paso a una múltiple confrontación de concepciones. ¿Cuáles? Agotado el fantasma de la concepción dominante se abre paso la discusión epistemológica en un nuevo encajonamiento: lo que Silverman entrevió como contraposición "paradigmática" va a encontrar eco en el debate que se plantea la dualidad de la convivencia o no de la multiplicidad paradigmática. El libro de Burrell y Morgan (publicado en 1979, y el texto más citado en el campo académico en la década de los ochenta) dará pié a la triple posibilidad que encajona la discusión epistemológica de inicios de la década de los noventa; triple posibilidad a la que nos referimos al inicio de esta sección.

Pero, esta misma década de los noventa se abrió con una muy interesante particularidad: el "descubrimiento" por parte del campo del estudio de organizaciones, del "debate" 'modernismo-postmodernismo'.

Me parece que es la falacia de este tal debate lo que interesa más profundamente a M. Reed. Tal supuesto "debate" es el último (?) ropaje para el encajonamiento. En consecuencia, encierra un inmenso peligro; el de mantener la posposición del debate epistemológico de fondo. Tal "debate" pasa a ser el responsable de la más profunda dicotomía que se presenta en la triple posibilidad: profundizar en el desorden del campo académico dejando paso franco a la visión acomodaticia y utilitarista (un modo del no-debate; 
"exceso de relativismo epistemológico") u optar, ora por la inconmensurabilidad paradigmática, ora por el predominio de la tradición académica ortodoxa (otro modo del no-debate: "exceso de polarización teorica") (Reed, 1993: 174). El mal entendido debate modernismo-postmodernismo permite optar por la primera posibilidad del no-debate. $Y$ ello porque tal "debate", en el contexto de quienes a él se refieren desde el campo del estudio organizacional, no es más que el reciente distraz del "eterno retorno" a la oposición a otra supuesta concepción dominante... ahora identificada como "modernismo" o "modernidad". En resumen, el inmenso peligro que encierra el "debate" 'modernismo-postmodernismo' es el de servir de asiento a una falsa escogencia que siempre conduce al no-debate y, simultáneamente, permite investir de enemigo a una 'modernidad' que no cuenta como tal enemigo.

\section{Replanteando el debate epistemológico de las teorías de organizaciones}

¿Es posible salir de este encajonamiento? La respuesta de Michael Reed es sí. Bajo las siguientes condiciones: 1) reinterpretar la consagrada historia del pensamiento organizativo; 2) postular un sentido para el campo académico del estudio del fenómeno organizacional que no se agote en el mero fenómeno y que de significado al devenir de las diversas preocupaciones intelectuales "serias" que de él se han ocupado.

Reed cree identificar en algunas contribuciones particulares, que navegan en las turbulentas aguas de la dispersión del campo académico, una especie de "actitud histórica" que supone el arraigo de ese campo en una tradición académica que no se limita al supuesto origen en los comienzos del siglo XX. El apego a esa tradición permite desvanecer la acendrada imagen del fantasma de la discontinuidad con una concepción dominante en el debate epistemológico. "En lugar de presumir rupturas irreparables con el pasado y discontinuidades fundamentales en orientación y enfoques, la teoría organizacional contemporánea parece estar redescubriendo y renovando sus conexiones con formas de análisis que tienen sus raices en una narrativa intelectual coherentemente articulada y en programas que continúan expresándose en temas y problemáticas recurrentes." (Reed 1993: 179).

¿En qué consiste la tradición y narrativa intelectual a la que hace referencia el autor?

Reed reinterpreta la historia del pensamiento sobre organizaciones tomando como punto de partida y como hilo conductor la reflexión -generalmente de corte sociológico - referida al papel que juega el fenómeno organizacional en la construcción del proceso de modernización de la sociedad. Como si siempre hubiese estado de fondo la hipótesis siguiente: "Las organizaciones burocráticas parecen proveer la exacta combinación de instrumentalidad cognoscitiva, anonimia moral y efectividad técnica que asegura los prerequisitos necesarios para la exitosa transición de un orden social basado en la costumbre y la tradición a uno fundado en el cálculo y el control racional". (Reed 1992: 2). 
¿Cómo procede operativamente?

En la investigación sobre el fenómeno organización han habido continuidades temáticas desde el propio origen de las reflexiones propias de la sociología, la economía y la filosofía politica (los temas esenciales son: racionalidad, alienación, anomia, poder).

De manera que puede interpretarse la concentración exclusiva en un tema o la aparente dispersión temática de subtemas en el desarrollo de los "enfoques" organizacionales como el otro lado de la moneda de la dispersión epistemológica. Esa dispersión temática lleva la responsabilidad de hacer aparecer como nuevos enfoques lo que no es más que un inconsciente, y hasta ingenuo, renacer de la indagación por un tema de vieja data.

De ese modo, los temas del presente, es decir de las temáticas últimas que ocupan la avanzada del desarrollo actual del análisis organizacional (vigilancia y control, poder profesional, políticas del diseño organizacional, racionalidad organizacional, organización postmoderna) se pueden entender como redescubrimientos de la continuidad temática (Reed 1993: 179).

La narrativa, entonces, se presenta reconstruida en base a la continuidad temática. Esta manera de contar la constitución del campo académico del análisis organizacional pugna, claro está, con las "tradicionales 'historias' del pensamiento organizativo", v. gr. las que ubican el origen de éste en el desarrollo de la investigación empiricista propia de comienzos de siglo en USA en relación con el rendimiento productivo del trabajo fabril.
$Y$, ¿qué hacer con esa nueva historia, esa nueva narrativa?

Ella engrana como pieza esencial en la proposición de Reed de reconstruir el campo académico del análisis organizacional como una "práctica intelectual". Esta es una proposición basada en la idea de práctica formulada por el filósofo Alasdair Macintyre: una práctica es "una forma coherente y compleja de actividad humana cooperativa, socialmente establecida, mediante la cual se realizan bienes internos a esa forma de actividad, en la medida en que se intentan alcanzar los patrones de excelencia que son apropiados para, y que definen parcialmente esa forma de actividad; ello conlleva al resultado de que los poderes humanos para alcanzar la excelencia, las concepciones de los fines y los bienes envueltos en la práctica mejoran sistemáticamente." (Maclntyre, 1981: 187), (Reed 1993: 176). La "práctica intelectual" que define al estudio de organizaciones tiene su propia historia oculta, por así decirlo, en la aparente dispersión de lo que, según el relato de Reed, ha sido la permanente y continua preocupación por generar el bien definido por la comprensión del proceso de constitución de la sociedad moderna a partir de la observación rigurosa del fenómeno organización.

iEsto constituye la proposición de un nuevo piso común para el debate epistemológicol

¿Cómo se plantea, entonces, el debate epistemológico?

En primer lugar, Reed sugiere la idea de proponer una noción de Organización, como constitutiva de una Teoría -entendida como "práctica intelec- 
tual"- que la define como una "práctica social": Prácticas sociales que se engranan para ensamblar e integrar otras prácticas sociales (las que transforman las condiciones materiales e ideales que hacen posible la acción colectiva) (Reed, 1992: 112-115).

En segundo lugar, esa práctica social -la Organización- es identificada, conceptualmente, según el esquema de Anthony Giddens sobre el surgimiento y sostenimiento de la modernidad (Giddens, 1991). La noción de Organización que se propone entonces como elemento nucleico para el desarrollo del campo académico entendido como una "práctica intelectual" no se refiere tanto a la distinción que hace especifica a una cierta organización particular; mucho menos, a la pretensión de descubrir los mecanismos de transformación que convierten la funcionalidad o papel de las organizaciones. Más bien se trata de observar, por intermedio de las organizaciones -o mejor, la Organización (como proceso societal)-, el devenir propio de la modernidad.

La noción de Organización que se propone está ligada indisolublemente a un rasgo general de la modernidad: la profusión de la organización. Como bien dice Giddens: "Lo que distingue a las organizaciones modernas no es tanto su tamaño o su carácter burocrático, como el monitoreo reflexivo y concentrado que dicho tamaño y carácter burocrático llevan consigo. Quien dice modernidad no dice justamente organizaciones sino organización; es decir, el control regularizado de las relaciones sociales a través de indefinidas 'distancias' espacio-temporales" (Giddens, 1991: 16).

\section{Conclusión}

Habíamos señalado al inicio que esta manera de entender y practicar el debate sobre el status de las teorias de organizaciones en términos de su fundamentación, tendría, al menos para el campo académico, implicaciones insoslayables. Se entiende, ahora que el debate contemporáneo más general lo hace manifiesto, que resulta una insensatez seguir paseando de torre en torre (de enfoque en enfoque) desentendiéndonos del problema de saber si lo que nos puede causar el vértigo es la lejanía del piso o, más bien, la permanente posposición de un debate que la academia, al menos, no puede eludir.

En consecuencia, esta proposición de estructuración del campo académico del estudio de organizaciones que hemos presentado en este trabajo intentaría diferenciarse, radicalmente, de todo cuanto se sugiere desde el campo productivo-comercial de las "tecnologías gerenciales" (Dávila, 1994). Sin embargo, este último campo representará para la "práctica intelectual" un riquísimo espacio discursivo que alimentará el sustancial análisis organizacional que, con profunda mirada crítico-histórica, produce el alerta imprescindible de esta época de transformación refinada de los tiempos modernos. Asi parece impostergable la tarea de una critica de lo que pudiera denominarse el "pensamiento" gerencial. 


\section{Referencias bibliográficas}

Burrell, Gibson and Morgan, Gareth (1979), Sociological Paradigms and Organisational Analysis, Heinemann, London.

Dávila, Jorge (1994), Crítica parcial de la "Calidad Total", Revista FACES Universidad de Carabobo, Año 4, $\mathrm{N}^{*}$ 10, pp. 20-37.

Donaldson, Lex (1985), In Defence of Organization Theory: A reply to the critics, Cambridge University Press, Cambridge.

Flood, Robert and Jackson, Michael (1992), Critical Systems Thinking. Directed Readings, Wiley, New York.

Giddens, Anthony (1991), Modernity and SelHdentity. Self and Society in the Late Modern Age, Polity Press, London.
Jackson, Michael (1992), Systems Methology for the Management Sciences, Plenum Press, London.

Macintyre, Alasdair (1981), After Virtue: A Study in Moral Theory, Duckworth, London.

Morgan, Gareth (1986), Images of Organizations, Sage, Beverly Hills.

Morgan, Gareth (1989), Creative Organization Theory. A Resourcebook, Sage, Beverly Hills.

Reed, Michael (1992), The Sociology of Organizations. Themes, Perspectives and Prospects, Hawester, New York.

Reed, Michael (1993), Organizations and Modernity: Continuity and Discontinuity in Organization Theory en Hassard, John and Parker, Martin (Eds.), Postmodernism and Organizations, Sage, London.

Silverman, David (1970), The Theory of Organizations, Heinemann, London. 